Revista de la red interuniversitaria de estudios sobre las literaturas rioplatenses contemporáneas en Francia

\title{
El imaginario apocalíptico en la literatura hispanoamericana: esbozo de una tipología
}

\author{
Geneviève Fabry
}

\section{OpenEdition}

\section{Journals}

Edición electrónica

URL: http://journals.openedition.org/lirico/689

DOI: $10.4000 /$ lirico.689

ISSN: 2262-8339

Editor

Réseau interuniversitaire d'étude des littératures contemporaines du Río de la Plata

\section{Referencia electrónica}

Geneviève Fabry, «El imaginario apocalíptico en la literatura hispanoamericana: esbozo de una

tipología », Cuadernos LIRICO [En línea], 7 | 2012, Puesto en línea el 11 octubre 2012, consultado el 19 abril 2019. URL : http://journals.openedition.org/lirico/689; DOI : 10.4000/lirico.689

Este documento fue generado automáticamente el 19 abril 2019.

\section{cc) $(1) \ominus$}

Cuadernos LIRICO está distribuido bajo una Licencia Creative Commons Atribución-NoComercialSinDerivar 4.0 Internacional. 


\title{
El imaginario apocalíptico en la literatura hispanoamericana: esbozo de una tipología
}

\author{
Geneviève Fabry
}

1 El mito fundacional del apocalipsis despliega un imaginario subyacente en muchas obras representativas de la literatura hispanoamericana de los siglos XX y XXI en general, y de la narrativa conosureña en particular. Este mito, que hunde sus raíces en una de las grandes fuentes de la cultura occidental, la Biblia, ha estado presente en la narrativa hispanoamericana desde sus inicios, ofreciendo una posibilidad de evocar y reformular el cataclismo que para los pueblos indígenas amerindios significó la conquista. Si nos atenemos al siglo XX, llama la atención el vigor con que se han puesto en escena avatares del imaginario apocalíptico, y la centralidad que éstos ocupan dentro del canon de la literatura hispanoamericana en general y del Cono Sur en particular, a pesar de la visible disparidad de sus manifestaciones. Este imaginario informa textos de autores argentinos tan protagónicos y diversos como Leopoldo Marechal, Eduardo Mallea, Roberto Arlt y Ernesto Sábato, si bien se le imprimen finalidades distintas - optimista en el caso de Marechal, desviando la simbología cristiana hacia un propósito laico o francamente anticristiano en los otros tres autores. La veta apocalíptica no pierde relevancia en la generación del boom, sino que, al contrario, algunos de sus textos clave -"Apocalipsis de Solentiname" de Julio Cortázar, La guerra del fin del mundo, de Mario Vargas Llosa, Cien años de soledad, de Gabriel García Márquez, Cristóbal Nonato de Carlos Fuentes- se nutren de ella.

2 Este breve vistazo destaca ya, por la heterogeneidad de estas primeras referencias, a la vez la riqueza y la complejidad de la tarea que supone la exploración e interpretación de este imaginario. Este ha sido el desafío que ha intentado enfrentar un equipo afincado en Bélgica. Su investigación ha desembocado en la publicación de un volumen colectivo, coeditado por Ilse Logie (U. Gante), Pablo Decock y Geneviève Fabry (U. Lovaina) : Los imaginarios apocalípticos en la literatura hispanoamericana contemporánea (Peter Lang, 2010). Las líneas que siguen retoman y prolongan los resultados de este trabajo colectivo. 
3 Para empezar, no parece inútil recordar algunas definiciones básicas : o sea, ¿en qué sentido manejamos el doble concepto de "imaginario apocalíptico" ? En el campo de los estudios literarios, ${ }^{1}$ la noción de imaginario remite a una red de representaciones mentales alimentadas por un legado mítico, religioso y/o histórico, dotada de un valor epistemológico y axiológico. Como precisa Maurice Godelier, "comme toute représentation est en même temps le produit d'une interprétation de ce qu'elle représente, l'imaginaire, c'est l'ensemble des interprétations que l'humanité a inventé pour s'expliquer l'ordre ou le désordre qui règne dans l'univers et pour en tirer des conséquences pour la manière dont les humains doivent organiser leur vie sociale" (598).

4 En cuanto al segundo término, "apocalíptico", sin olvidar las fundamentales aportaciones de las culturas indígenas de América Latina al respecto, lo situamos sobre todo en el contexto global de las culturales occidentales que conciben esencialmente el tiempo de manera lineal, en tensión entre un principio y un final. A una creación ex nihilo se opondría el final del mundo, concebido al mismo tiempo como destrucción del orden antiguo y revelación de las verdades esenciales del mundo y del hombre. Recordemos aquí la doble significación de "apocalipsis". El término griego remite en efecto al sentido general de "revelación", esto es, la acción de desvelar lo oculto y lo secreto. La visión apocalíptica está íntimamente ligada a la idea judaica del mesianismo, ya que se proyecta hacia un futuro en el que se resolverá la historia. Más tarde será heredada por el cristianismo, donde alcanza su desarrollo pleno entre el segundo siglo antes de Cristo y el segundo después de Cristo. En la tradición judeo-cristiana del género apocalíptico (véanse los textos del Primer Testamento, como el libro de Daniel, así como los del Segundo Testamento: partes de los evangelios sinópticos y el Apocalipsis de san Juan), el contenido de esta revelación atañe fundamentalmente a cuestiones escatológicas. El mito del Apocalipsis, en las variantes más fieles, remite a la revelación profética de un acontecimiento dramático para la humanidad, en el que las fuerzas del mal vencen a las del bien en un gran cataclismo cósmico, después del cual Dios destruye los poderes dominantes para instaurar la supremacía del bien alcanzándose así el fin de los tiempos.

5 Un breve repaso por una serie de publicaciones recientes procedentes de campos tan disímiles como la historia, la filosofía y la ecología da cuenta de la presencia de múltiples avatares del mito apocalíptico y de sus ambigüedades en los discursos sociales y culturales actuales. Quizás sea en el último campo evocado donde la referencia apocalíptica sea hoy la más presente y visible, pero el fin del mundo por colapso medioambiental es hoy en día un tema tan traído y llevado que quizá no valga la pena insistir demasiado en ello. La magistral síntesis Apocalypses y millénarismes del historiador Eugen Weber nos recuerda que tal obsesión "apocalíptica" no es nueva y traza los contornos de la permanencia de los esquemas apocalípticos y milenaristas (siendo éstos la versión inmanente, inminente y regeneradora -Weber 39- de aquéllos) en la historia occidental desde el comienzo de la era cristiana hasta la actualidad.

6 La novedad que aporta el siglo XX al respecto es la radicalización de la conciencia de un apocalipsis posible y efectivo a partir de la experiencia traumática de las dos guerras mundiales, especialmente la segunda. El mal radical se encarna ahora en los mecanismos de un totalitarismo destructor que sella el final de una cierta concepción de la humanidad y su "progreso". Si, como quiere Agamben, el campo de concentración es la expresión más definitiva de la modernidad, los tiempos posteriores abren la era de los supervivientes, los que tienen conciencia de vivir después de la catástrofe, o sea after the end (como reza el título de Berger), o en tiempos postapocalípticos, según el término acuñado por el 
mejicano Monsiváis. Lo que puede resultar interesante recalcar aquí es que las ideologías destructoras se refieren una vez más al apocalipsis para justificarse, desde el nazismo (como lo demuestra Philippe Burrin) hasta los movimientos terroristas actuales (véase el estudio de Yves Bourdillon).

7 En el campo filosófico, varios pensadores actuales están reflexionando acerca de las condiciones que permiten pensar el riesgo y la catástrofe, articular racionalmente la acción presente y la predicción del porvenir. Quizás sea el pensador francés y catedrático en Stanford Jean-Pierre Dupuy, el que con mayor agudeza y claridad haya desarrollado estos temas en una serie de publicaciones importantes, muy inspiradas en el pensamiento de René Girard y de Hans Jonas. Según Dupuy, en su libro Pour un catastrophisme éclairé, ha llegado el momento de "llevar a cabo una reflexión acerca del destino apocalíptico de la humanidad" (contratapa). En efecto :

Tout nous porte à penser que nous ne pouvons étendre indéfiniment, ni dans le temps ni dans l'espace, le mode de développement qui est le nôtre. Mais remettre en cause ce que nous avons appris à assimiler au progrès aurait des répercussions si phénoménales que nous ne croyons pas ce que nous savons pourtant être le cas. Il n'y a pas d'incertitude ici, ou si peu. [...] [C]'est non seulement le savoir qui est impuissant à fonder la crédibilité, mais c'est aussi la capacité de se représenter le mal, ainsi que la mobilisation de tous les affects appropriés (144-145, subrayado nuestro).

8 Para reaccionar adecuadamente a las amenazas que ensombrecen el porvenir de la humanidad, la primera necesidad sería pues la de la creencia en la realidad del peligro. Esta no estriba en el saber sino en una compleja red de representaciones mentales con la carga emocional y afectiva asociada, especialmente, dice Dupuy, las que están relacionadas con la problemática del fin y del mal. ¿No nos toparíamos aquí con una evocación del imaginario apocalíptico ? De hecho, la psicología cognitiva nos enseña que nuestra acción es menos regida por nuestro saber que por nuestras convicciones, menos por nuestros conocimientos que por nuestras creencias. ¿Qué necesita la creencia para construirse? Una serie de elementos factuales, es cierto. Pero estos elementos sólo cobran sentido si vienen articulados en una forma significativa, es decir unos símbolos y un relato, que impliquen la afectividad y la imaginación, con sus distintos estratos conscientes e inconscientes. Necesitamos la mediación de la forma artística para inscribir nuestros conocimientos, incluso los menos precarios, en la historia y la acción colectivas. Estudiar esta mediación y conectarla con las inquietudes de nuestros tiempos quizá constituya una de las tareas más importantes de la crítica literaria, y el estudio del imaginario apocalíptico en la literatura hispanoamericana, uno de los case studies más apasionante y relevante que se pueda imaginar. ¿Cuáles son las enseñanzas de una exploración de textos significativos de la literatura hispanoamericana desde esta perspectiva?

9 En primer lugar, se ha podido comprobar que el imaginario no es un cajón de sastre sino que, manejado con el debido rigor, es una auténtica categoría epistemológica, conlleva un saber acerca de la situación del hombre en el mundo y en la Historia. De manera más precisa, podemos añadir que este imaginario es constituido ante todo, en el caso del imaginario apocalíptico, no tanto por un contenido (las famosas representaciones mentales compartidas) como por el modo alegórico de leer la Biblia: los símbolos más recurrentes del libro bíblico figuran acontecimientos catastróficos de la Historia reciente. Por alegoría, por supuesto, no se remite solo a la estrecha concepción clásica : "relación convencional entre una imagen ilustrativa y un sentimiento abstracto" (Avelar 15). Más 
fundamentalmente, la alegoría se refiere a la "historia sufriente del mundo" (Benjamin citado por Avelar, 14), una historia cuya interpretación sigue siendo tributaria de esquemas religiosos que se han vuelto lejanos pero no han desaparecido por completo. Según Avelar, "[con el barroco], [1]a alegoría florece en un mundo abandonado por los dioses, mundo que, sin embargo, conserva la memoria de ese abandono y no se ha rendido todavía al olvido" (18). La alegorización persistente de la historia latinoamericana basada en mitemas apocalípticos es un fenómeno general, como apunta Lois Parkinson Zamora en su libro pionero. Todos los textos que exhiben o esconden una referencia al mito apocalíptico interpretan las crisis históricas a la luz de la gran matriz de sentido que es el texto bíblico entendido de manera esquemática. En este sentido, estos textos manifiestan huellas de una fijación del sentido en la primera etapa de la lectura, lo que Jean-Louis Dufays llama el "clichage" del texto (1994:158), que equivale muchas veces, en el caso del último libro bíblico, a una alegorización. Pero muchos textos también van más allá de esta primera etapa de la lectura del texto bíblico. Como subraya Dufays, "au stéréotypage succède fréquemment un acte de suspension, un moment où l'on choisit de renoncer à la clôture du sens et à la fonction référentielle pour valoriser la dimension archétypale et la polysémie des signes" (Dufays $2006: 5$ ). La diferenciación entre varias modalidades de lectura alegórica tiene que ver con la fuerza de este acto de suspensión y sus huellas que se superponen y hacen de las reescrituras posteriores palimpsestos de lecturas.

10 En segundo lugar, la exploración en múltiples textos y tradiciones ha confirmado la gran heterogeneidad de las plasmaciones de "lo" apocalíptico. Si bien se ha identificado el género apocalíptico bíblico (destacando más específicamente los mitemas del Apocalipsis de Juan) -y su lectura alegórica- como fuente principal del imaginario occidental del mismo nombre, ¿qué es lo que queda de él en las versiones literarias hispanoamericanas contemporáneas ??

11 La respuesta a esta pregunta es matizada y puede desplegarse bajo la forma de una tipología gradual. Esta tipología intenta distinguir categorías caracterizadas a la vez por el qué de la reescritura apocalíptica y el cómo (¿cómo se ha leído el mito/os mitos originales para llegar a esta apropiación ?). Hemos llegado así a la determinación de cuatro categorías principales que apuntan a la re-figuración ${ }^{3}$ del mito apocalíptico. El término de refiguración hace énfasis en la dimensión de repetición y reinscripción de un hipotexto más o menos difuso. Estas categorías son las siguientes :

- refiguración mítica explícita ;

- refiguración mítica implícita;

- refiguración estereotipada ;

- refiguración postapocalíptica.

12 Primero, están las obras en las que el intertexto bíblico está presente de manera significativa y explícita. Lo que se retoma de la gran matriz bíblica es a la vez su sintaxis (tensión dinámica entre génesis y apocalisis) y su léxico (el uso más o menos descontextualizado pero reconocible de sus símbolos más llamativos, ya no ordenados en series significantes). Es llamativa la concentración de textos poéticos en esta primera categoría : desde "Pax" de Darío o Fin de mundo de Neruda hasta ciertos textos de Pacheco, Aridjis y Sara de Ibáñez, la poesía es un discurso especialmente apto para recoger los ecos del cuestionamiento metafísico de un sujeto diversamente expuesto a las crisis de la modernidad (desde la primera guerra mundial hasta el colapso medioambiental) y plasmar sus esperanzas y desconciertos en un discurso polifónico en el que el intertexto bíblico resalta ora la profecía, ora la nostalgia, pero casi siempre cargado de un 
reconocido simbolismo. En la narrativa también, aunque falte en general una expresión directa de ese trasfondo metafísico, se observa un trabajo intertextual preciso con varios textos bíblicos, especialmente el texto final del Segundo testamento. Este trabajo intertextual puede atañer a los personajes (por ejemplo la descripción de Antonio el Consejero en La guerra del fin del mundo de M. Vargas Llosa, del narrador en La virgen de los sicarios de F. Vallejo o de la equívoca iniciación del sacerdote de Nocturno de Chile de R. Bolaño), o a la transposición de uno de los mitemas fundamentales (el juicio final en $\mathrm{El}$ asalto de Arenas o en Redoble por Rancas de Scorza ; la descripción cataclísmica del fin del mundo, revelador de las condiciones de la escritura, como en El sueño de Santa María de las piedras de Méndez, uno entre tantos vástagos de Cien años de soledad, prototípico en este sentido). En todos estos casos, la inscripción del imaginario apocalíptico no sólo es explícita sino que también es significativa : se ofrece en general como entrada de un doble sentido posible; el léxico (referencias simbólicas o textuales) no se puede aislar de una sintaxis (a nivel macroestructural, la referencia apocalíptica orienta el sentido de los fines narrativos y los comienzos diegéticos). La guerra del fin del mundo, paradigmática en muchos sentidos, no solo presenta un texto esmaltado de referencias intertextuales bíblicas para describir un mundo que es la presa de fanatismos encontrados. Además, su manera de descifrar las relaciones entre sagrado y violencia traduce precisamente uno de los resortes del saber antropológico de la Biblia y más concretamente, del Segundo Testamento, a saber la imposibilidad de eliminar la violencia por la expulsión del chivo expiatorio disfrazado de culpable de la crisis.

13 Esta relevancia macroestructural del imaginario apocalíptico se encuentra también en nuestra segunda categoría de textos pero debilitada por la desvinculación entre sintaxis y léxico del mito apocalíptico, este último encontrándose más aludido que citado. En esta segunda categoría, encontramos básicamente dos tipos de textos. Primero, están los textos en los que la referencia al mito apocalíptico se encuentra reducida a la matriz narrativa/teleológica (la sintaxis sin el léxico). En segundo lugar, la refiguración mítica puede apoyarse en una referencia difusa, pero significativa, de símbolos de raigambre apocalítica desvinculados del mito de origen (el léxico sin la sintaxis). Del primer tipo es representativo un texto como "Apocalipsis en Solentiname". Aparte de la mención en el título, el texto carece de un trabajo intertextual con la Biblia. Sin embargo, la elaboración cuidadosa de las isotopías entretejidas de la revelación (fotográfica/política) y de la destrucción apunta una vez más al gran generador de tramas que es el "código" bíblico ( $c f$. N. Frye). Del segundo tipo, nos parece muy ilustrativa la obra póstuma de Bolaño : 2666. El título alude a la cifra de la Bestia, pero, al contrario de lo que ocurre en otros textos de Bolaño (como Nocturno de Chile), aquí el Apocalipsis de Juan no es objeto de reescritura, aunque sea en clave paródica. El título enfatiza la figuración del Mal de la que la novela ofrece una geneología que, a pesar de su inscripción en la historia y la geografía, se niega a desplegar una temporalidad lineal. La imbricación de las cinco novelas ("Partes") pone en tela de juicio la idea misma de una catástrofe final que pudiera tener dimensiones regeneradoras, como asimismo niega al acto narrativo una dimensión teleológica : en este sentido 2666 es un anti-Cien años de soledad.

14 Mientras que la refiguración mítica se fundamenta en un uso consciente del simbolismo apocalíptico, este se ve reducido a mera estereotipia en la tercera categoría de textos estudiados. La ambigüedad interpretativa se reduce drásticamente mientras que el cuestionamiento metafísico tiende a desaparecer. No siempre es fácil discernir entre inscripción mítica o estereotípica de un motivo. En esta tercera categoría, es la polisemia 
de la lectura de la Biblia, la suspensión del "clichage" (para retomar la expresión de Dufays) la que plantea problema. Veamos por ejemplo dos textos poéticos de los años 60 para intentar destacar la diferencia entre refiguración mítica y estereotipada: Fin de mundo de Neruda y Apocalipsis de Cardenal. El poemario Fin de mundo (1969) de Pablo Neruda destaca por su apropiación del mito apocalíptico sobre todo a nivel de la dispositio. Se puede observar ante todo la macroestructura de una historia lineal polarizada entre un comienzo y un final encarnado en la "Bomba", "la usina total de la muerte" (414), a la que Neruda dedica dos poemas (414-431). Sin embargo, las alusiones bíblicas en este libro no se pueden considerar como estereotipos : se alejan de la estructura fija del sintagma o del motivo y mantienen una ambigüedad semántica ajena al esquematismo del estereotipo. De hecho, no falta en el libro una serie de alusiones bíblicas, desde Goliat (410), hasta la crucifixión (358) y la resurrección, pasando por "nuestro pan de cada día" (359). Pero lo que llama la atención es la dinamización y la complejificación de los motivos apocalípticos. Quisiera dar de esto un solo ejemplo : se trata de la posición del YO lírico. Es sabido que el escriptor del relato del Apocalipsis es un vidente (Apoc I, 11-12) que nos entrega visiones exclusivas. Desde el punto de vista secuencial, las diferentes partes del poemario ofrecen al lector verdaderas visiones del planeta dolido y de su historia a lo largo del siglo XX. Pero el YO nerudiano canta y emerge en el seno de un "nosotros", no es un vidente. Así empieza el libro :

Qué siglo más permanente!

Preguntamos:

Cuándo caerá ? Cuándo se irá de bruces

al compacto, al vacío?

$[\ldots]$

Cuando cayó la Bomba

[...]

pensamos irnos con el atadito, cambiar de astro y de raza.

[...] Era grave

esta vergüenza

de ser hombres

iguales

al desintegrador y al calcinado. (357)

El YO se define como superviviente y testigo ("Esto pasó. Yo lo atestiguo" 371), a la vez víctima y "cómplice de la humanidad/con mis hermanos asesinos" (464). No hay aquí (aunque sí en otras partes de la obra nerudiana) maniqueísmo en la determinación de las responsabilidades de la catástrofe, al mismo tiempo humana, ecológica y poética, que el poeta evoca. El estereotipaje que siempre tiende a estigmatizar la postura ajena no puede ser operatorio aquí. ${ }^{4}$

16 Esta es, me parece, la mayor diferencia que podemos observar si comparamos el tratamiento de la catástrofe nuclear en Neruda y Cardenal. En "Apocalipsis" (1965), la posición del vidente es asumida desde la certidumbre de la verdad y la convicción de formar parte del bando de los salvados. Así rezan los primeros versos :

Y HE AQUÍ

QUE VI A UN ÁNGEL

(TODAS SUS CÉLULAS ERAN OJOS ELECTRÓNICOS)

Y OÍ UNA VOZ SUPERSÓNICA

QUE ME DIJO : ABRE TU MÁQUINA DE ESCRIBIR Y ESCRIBE. 
Y VI COMO UN PROYECTIL PLATEADO QUE VOLABA

Y DE EUROPA A AMÉRICA LLEGÓ EN 20 MINUTOS

Y EL NOMBRE DEL PROYECTIL ERA BOMBA H

(Y EL INFIERNO LO ACOMPAÑABA) (CARDENAL : 118) telecomunicación y de la muerte masificada destruye el simbolismo inherente a las visiones apocalípticas. La sistematización de la traducción alegórica de los símbolos en un plano ideológico unívoco empobrece drásticamente el simbolismo bíblico hasta destruirlo. Nueva York es la nueva "Babilonia" azotada por un justo castigo. ${ }^{5}$

vez destruido el simbolismo por el mecanismo repetido de la traducción alegórica fuertemente ideologizada, el motivo bíblico del ángel que abre el libro de las visiones desemboca, como lo nota Julio Ortega, en el humor : "este es un ángel didáctico, que le paga al glosador con un cheque sin fondos" (Ortega: 60). Leído hoy, este texto parece profético en su anticipación tanto de ground cero (ibid.) como del desplome de la Bolsa. Pero lo que nos importa recalcar es que el vidente no tiene ninguna responsabilidad en lo que acaece : el maniqueísmo de la visión pertenece al ángel "dictador" (quien le dicta lo que debe ver, escuchar y escribir). ${ }^{6} \mathrm{Si}$ el imaginario apocalíptico aparece en Cardenal como estereotipado no es porque podamos encontrar estereotipos relativos al imaginario apocalíptico: los lexemas típicos desde este punto de vista son citas textuales de una fuente identificada, relacionadas con la serie más previsible de los motivos bíblicos (el ángel, la Prostituta, la Bestia, el Cordero, las copas, etc.). Lo que es estereotipado, en el caso de Cardenal, es ante todo su propia lectura del libro bíblico. Cardenal se limita a un "clichage" (Dufays $1994: 158$ ) determinado por presupuestos antiimperialistas, anticapitalistas y antiestadounidenses. Estos presupuestos forman ideologemas compartidos por el discurso revolucionario latinoamericano de los años 60 , tanto en el ámbito político como en el poético. Son estos ideologemas, verdaderas estereotipías caracterizadas no solo por su carga ideológica y su carácter difuso, sino también por su "significación abstracta y esquemática" (Dufays 1994: 57), los que el lector de Cardenal está invitado a reconocer y adoptar.

En textos más recientes, sobre todo narrativos, podemos reconocer los avatares de una lectura más bien reductora del mito apocalíptico. Al rumor de las cigüeñas de Gabriela Ovando podría ser un caso representativo de la refiguración estereotipada del mito apocalíptico. Todos los ingredientes del mito apocalíptico están presentes de manera explícita pero la polisemia del mito está ausente. El diluvio final aparece como un remake del cierre de Cien años de soledad, sin que se encuentre una verdadera justificación simbólica o estructural inherente a la novela misma. A primera vista, las novelas de Guebel (Los elementales) o de Aira ( $L a$ villa, La prueba) parecen presentar las mismas características, pero a través de un mayor distanciamiento en la enunciación narrativa. Sin embargo, su rasgo verdaderamente notable es otro : ponen en tela de juicio, por su cultivo del non sense y del absurdo, la posibilidad misma del gesto hermenéutico que subyace en las reescrituras no paródicas del mito apocalíptico. Esta imposibilidad también puede aparecer en textos enunciados en clave seria : sería el caso de la novela de Eltit ( Jamás el fuego nunca) cuyos personajes casi beckettianos parecen asistir no sólo al derrumbe del ideal revolucionario sino también al ocaso de la palabra que permitía articular utopías y vivencias. Además, la insistente referencia a los medios masivos de comunicación en los dos relatos de Aira hace intervenir también otro parámetro en el establecimiento de la refiguración como estereotipada. El proceso de filtro, reproducción,

Cuadernos LIRICO, 7 | 2012 
diseminación operado por los medios, especialmente la televisión y sus reality shows, está puesto de relieve en algunas obras del Nuevo Cine Argentino, como en La ciudad de María de Bellande, documental en el que lo apocalíptico apenas sobrevive bajo los nuevos disfraces de un milenarismo reciclado por la televisión que todo lo transforma en objeto de consumo visual.

20 En la cuarta y última categoría, tendríamos textos provistos de una referencia al mito apocalíptico que solo conserva, aislado, un mitema truncado : el de una catástrofe de gran magnitud. Esta catástrofe, amén de coincidir con muchas de las mitologías indoamericanas y de la experiencia de la Conquista como trauma colectivo, se concibe como apocalipsis por el salto cualitativo radical que impone. Este salto en sí no resulta pensable; en consecuencia, el énfasis cambia de lugar y lo que una serie significativa de obras narrativas de las últimas décadas se dedican a imaginar es lo que pasa después del fin , según la acertada y oximórica expresión de James Berger. De ahí el rótulo "postapocalíptico" que define textos que operan en esta zona fronteriza abierta por el trauma. De ahí también la noción más conceptual que estrictamente temporal del término, como explica Carlos Monsiváis en Rituales del caos. Típicos de esta refiguración postapocalíptica serían los textos de Marcelo Cohen (Donde yo no estaba, La ilusión monarca ) o la novela de Mairal, El año del desierto. Esta pone en marcha un tiempo regresivo que diluye los adelantos de la modernidad, revelando las aporías del modelo en su versión neoliberal argentina pero destacando también un resto no deleznable: lo único que sobrevive al desastre es la palabra que surge después de la mudez y el olvido.

21 Por supuesto, como toda tipología, esta tiene un interés conceptual y pedagógico, pero es cierto que presenta amplias zonas de solapamiento: las categorías son porosas y los textos literarios en su labilidad rehúsan a encerrarse en una casilla única. Un solo ejemplo mostrará la relatividad de las categorías que hemos intentado definir. 2666, hemos dicho, es una refiguración mítica implícita, si dejamos de lado la clara alusión del título. Obviamente, sin embargo, las raíces del feminicidio que describe despiadadamente la "Parte de los crímenes", se hunden en la Historia occidental y más concretamente en la Shoah. Las trayectorias cruzadas de los personajes en el vasto fresco diseñado por Bolaño apuntan a ese agujero negro de la humanidad en el que un umbral moral se ha franqueado de manera irreversible. En este sentido, 2666 es también indudablemente una gran fábula postapocalíptica. Escribimos -y leemos- todos después de Auschwitz, después de haber comprobado entre atónitos y vergonzosos, el fin de cierta idea de lo humano.

\section{BIBLIOGRAFÍA}

Avelar, Idelber. Alegorías de la derrota : la ficción postdictatorial y el trabajo del duelo. Santiago :

Editorial Cuarto Propio, 2000.

Berger, James. After the End. Representations of post-apocalypse. Minneapolis/London : University of Minnesota Press, 1999. 
Bourdillon, Yves. Terrorisme de l'apocalypse. Enquête sur les idéologies de destruction massive. Paris : Ellipses, 2007.

Burrin, Philippe. Ressentiment et apocalypse. Essai sur l'antisémitisme nazi. Paris : Seuil, 2004.

Cardenal, Ernesto. Poesía, sel. y prólogo de C. Vinthier. La Habana : Casa de las Américas, 1979.

Darío, Rubén. Obras completas I. Poesía, ed. de J. Ortega con la colaboración de N. Vélez. Barcelona : Galaxia Gutenberg, 2007.

Decock, Pablo, Las figuras paradójicas de César Aira. Un estudio semiótico y axiológico de la estereotipia, (Thèse de doctorat, Université catholique de Louvain, Louvain-la-Neuve, 2009).

Deproost, Paul-Augustin, van Ypersele, Laurence, Watthee-Delmotte, Myriam, "Archétype, mythe, stéréotype : pour une clarification terminologique", in Mémoire et identité. Parcours dans l'imaginaire occidental. Louvain-La-Neuve : Presses universitaires de Louvain, 2008. 17-54.

Dufays, Jean-Louis. Stéréotype et lecture. Liège : Mardaga, 1994.

Dufays, Jean-Louis. "Stéréotyper, suspendre, rouvrir : le chantier sans fin de la lecture et de l'apprentissage" (2006). Texto comunicado directamente por el autor.

Dupuy, Jean-Pierre. Pour un catastrophime éclairé. Quand l'impossible est certain. Paris : Seuil, 2002.

Fabry, Geneviève, Ilse Logie y Pablo Decock. Los imaginarios apocalípticos en la literatura

hispanoamericana contemporánea. Oxford : Peter Lang, 2010.

Girard, René. Je vois Satan tomber comme l'éclair. Paris : Grasset, 1999.

Godelier, Maurice. "Imaginaire et symbolique". Le dictionnaire des sciences humaines, Sylvie Mesure et Patrick Savidan (dir.). Paris : PUF, 2006. 598-600.

Monsivais, Carlos. Los rituales del caos. México : Era, 1995.

Neruda, Pablo, Obras III, Buenos Aires, Losada, 1993.

Ortega, Julio, "La alegoría del apocalipsis en la literatura hispanoamericana", Decock P., Fabry G., Logie, I. (eds.). Los imaginarios apocalípticos en la literatura hispanoamericana contemporánea. Oxford : Peter Lang, 2010. 53-66.

Parkinson Zamora, Lois. Narrar el apocalipsis. La visión histórica en la literatura estadounidense y latinoamericana contemporánea, [1989], México, Fondo de Cultura Económica, 1994.

Raphaël, Freddy, "Esquisse d'une typologie de l'apocalypse", en L'apocalyptique, F. Raphaël et al. (ed.), Paris, Librairie orientaliste Paul Geuthner, 1977, 11-38.

Weber, Eugen. Apocalypses et millénarismes, Paris, Fayard, 1999.

\section{NOTAS}

1. Dejamos aquí de lado la tripartición lacaniana entre lo real, lo simbólico y lo imaginario. Remitimos en cambio a los trabajos de Durand, Jung, Bachelard, Burgos, Chelebourg, para limitarnos a los más importantes.

2. Para contestar a esta pregunta de investigación, nos han sido especialmente útiles los artículos de Marco Kunz, Julio Ortega, Milagros Ezquerro, Hervé Le Corre, Niall Binns, Norah Dei Cas Giraldi, Brigitte Adriaenssen, María Semilla Durán y Lucero de Vivanco, incluidos en el volumen colectivo ya mencionado (Fabry-Logie-Decock). 
3. Este término de 're-figuración' insiste sobre el papel fundamental que desempeña la figura, según Gervais, en el imaginario : 'L'imaginaire est l'interface par laquelle un sujet a accès aux éléments de la culture et se les approprie. Il est une médiation dont le travail transparait dans des figures' (Gervais 35). Citado por P. Decock en su tesis.

4. "sur le plan éthique[...] c'est un signe injuste, discriminatoire, violent à l'égard des réalités et des personnes à qui on l'applique. Le stéréotype apparaît ainsi comme un acte insupportable, car usé, mensonger, immoral, mais il faut insister sur le fait que ces reproches concernent le stéréotypage des autres [...]" (Dufays $2006: 3$ ).

5. "Y dijo el ángel : ¿Reconoces dónde estuvo Colombus Circle?

¿Y dónde estuvo el edificio de las Naciones UNidas?

Y donde estuvo Colombus Circle

yo sólo vi un hoyo en que cabía un edificio de 50 pisos

[...] CAYÓ LA GRAN BABILONIA

[...]

Y el ángel me dio un cheque del National City Bank

y me dijo : Cambia este cheque

y en ningún banco lo pude cambiar porque todos los bancos habían quebrado" (Cardenal:

121-122)

6. "Me dijo : Las naciones del mundo están divididas en 2 bloques

Gog y Magog-

pero los dos bloques son en realidad un solo bloque

(que está contra el Cordero)

y caerá fuego del cielo y los devorará

Y vi en la biología de la Tierra una nueva Evolución

[...]" (Cardenal : 123)

\section{RESÚMENES}

El artículo siguiente intenta esbozar los contornos del imaginario apocalíptico tal y como se plasma en la literatura hispanoamericana de los siglos XX y XXI, basándose en los resultados de un trabajo de investigación reciente (coeditado por Ilse Logie, Pablo Decock y Geneviève Fabry, Los imaginarios apocalípticos en la literatura hispanoamericana contemporánea, Peter Lang, 2010). La exploración en múltiples textos y tradiciones confirma la gran heterogeneidad de las plasmaciones de 'lo' apocalíptico. Si bien se ha identificado el género apocalíptico bíblico (destacando más específicamente los mitemas del Apocalipsis de Juan) -y su lectura alegóricacomo fuente principal del imaginario occidental del mismo nombre, ¿qué es lo que queda de él en las versiones literarias hispanoamericanas contemporáneas? A esta pregunta se enfrenta el artículo : distingue entre cuatro tipos de re-figuración del mito apocalíptico, comentando algunos ejemplos para cada categoría.

A partir d'un projet de recherche récent (coédité par Ilse Logie, Pablo Decock et Geneviève Fabry, Los imaginarios apocalípticos en la literatura hispanoamericana contemporánea, Peter Lang, 2010), l'article essaie d'ébaucher les contours de l'imaginaire apocalyptique, tel qu'on peut l'observer dans la littérature hispano-américaine des XXe et XXIe siècles. Un parcours rapide d'une série de textes et de traditions multiples confirme la grande hétérogénéité des représentations de 
l'apocalyptique "en lui-même". Bien qu'il soit possible d'identifier le genre apocalyptique biblique (en soulignant tout particulièrement les mythèmes de l'Apocalypse de Jean) -et sa lecture allégorique- en tant que source principale de l'imaginaire occidental sur la question, qu'en reste-t-il dans les versions littéraires hispano-américaines contemporaines ? L'article tente de répondre à cette question, en distinguant quatre types de re-figuration du mythe apocalyptique et en commentant quelques exemples pour chaque catégorie.

The following article attempts to outline the contours of the apocalyptic imagination as reflected in the American literature of the XX and XXI centuries, based on the results of a recent research project (co-edited by Ilse Logie, Paul Decock and Geneviève Fabry Los imaginarios apocalípticos en la literatura hispanoamericana contemporánea, Peter Lang, 2010). The exploration in multiple texts and traditions confirms the heterogeneity of

the image of 'the' apocalyptic. The Biblical apocalyptic genre (more specifically highlighting mythemes of Saint John's Revelation) and its allegorical reading have been identified as the main source of Western imagery of the same name, but what is left of it in contemporary Spanish American literary versions? The article tackles this question: it distinguishes between four types of re-figuration of apocalyptic myth, discussing some examples for each category.

\section{ÍNDICE}

Mots-clés: imaginaire, apocalypse, littérature hispano-américaine, allégorie, typologie

Keywords: imagination, apocalypse, spanish american literature, allegory, typology

Palabras claves: imaginario, apocalipsis, literatura hispanoamericana, alegoría, tipología

\section{AUTOR \\ GENEVIĖVE FABRY}

U.C. Louvain, Belgique 